\title{
Evidence of hemispheric specialization in marmosets (Callithrix penicillata) using tympanic membrane thermometry
}

\section{Tomaz, M.S. Verburg, V. Boere, T.F. Pianta and M. Belo}

Centro de Primatologia and Departamento de Ciências Fisiológicas, Instituto de Biologia, Universidade de Brasília, Brasília, DF, Brasil

\section{Correspondence \\ C. Tomaz \\ Departamento de Ciências Fisiológicas Instituto de Biologia, UnB \\ Brasília, DF \\ Brasil \\ Fax: +55-61-274-1251 \\ E-mail: ctomaz@unb.br}

Research partially supported by FINATEC. M.S. Verburg and T.F. Pianta were the recipients of fellowships from PIBIC/UnB/CNPq.

$V$. Boere was the recipient of a doctoral fellowship from CAPES

Received April 2, 2002 Accepted February 25, 2003

\begin{abstract}
Recent studies have employed tympanic thermometry to assess lateralization of cognitive and emotional functions in primates. However, no studies using this technique have investigated the possibility of hemispheric specialization in New World monkeys. Therefore, the aim of the present study was to investigate tympanic membrane (TM) temperature asymmetries and their possible correlation with stress responses in marmosets (Callithrix penicillata). Infrared TM thermometry was completed bilaterally in 24 animals (14 males and 10 females) during a stressful situation of capture and restraint. There were no significant differences between gender. A significant negative correlation was observed between TM temperature of the right ear and the number of captures $(r=-0.633 ; \mathrm{P}<0.001)$. Subjects with a more frequent previous history of captures ( 5 to 9 captures; $\mathrm{N}=11$ ) showed lower TM temperature when compared to those with fewer previous captures ( 1 to 4 captures; $N=13$ ). No differences were observed for the left TM temperature. These results suggest that under intense emotional challenge (capture and restraint) there is a stronger activation of the neural structures situated in the right brain hemisphere. Taken together, the data reveal for the first time evidence of hemispheric specialization in emotional physiological processing in a New World monkey.
\end{abstract}

\section{Introduction}

Most studies of lateral cerebral specialization in nonhuman primates have focused on handedness $(1,2)$ or use of primarily one part of the body (3). However, investigations of functional asymmetries based on behavioral responses have provided contradictory results. This is partially due to the diversity of procedures used, which many times makes it difficult to repeat the results, and to the fact that some tasks can recruit neural pathways of both hemispheres, confusing the interpretation of the results in terms of hemispheric specialization (4).

Recent studies, however, have employed tympanic thermometry, a noninvasive technique, to investigate lateralization of cognitive and emotional functions in human and nonhuman primates (5-9). It has been shown that human volunteers present a low tympanic membrane (TM) temperature in the 
left ear during verbal tasks and in the right ear during spatial tasks (7). Hopkins and Fowler (6) noticed the same aspect in chimpanzees (Pan troglodytes) during execution of different cognitive tasks. An increase in left TM temperature and a decrease in right TM temperature were found for matchingto-sample and visual-spatial discrimination tasks, respectively. No significant lateralized changes were observed during execution of motor control tasks. Recently, Parr and Hopkins (8) assessed the TM temperature of chimpanzees as the subjects watched emotional videos depicting socially positive, socially negative and neutral events and found increases in right $\mathrm{TM}$ temperature during the negative emotion condition.

Boyce et al. (5) showed that TM temperature could be also considered to be an indicator of biobehavioral stress responses. Significant asymmetries were observed in TM temperatures in rhesus macaques and humans, in which higher-magnitude left-to-right temperature gradients were associated with high frequency of locomotion in macaques and with lower resilience in humans.

To our knowledge, there are no studies in Neotropical primates testing a possible correlation between tympanic temperature and stress response. Since a difference in TM temperature is considered to be a physiological index of cerebral hemispheric activity, the results of such study could indicate a hemispheric specialization in New World primates. Therefore, the purpose of the present study was to assess TM temperature in marmosets (Callithrix penicillata), New World anthropoids, during a stressful situation of capture and restraint.

\section{Material and Methods}

\section{Subjects}

Twenty-four marmosets (Callithrix penicillata), 14 males and 10 females, served as subjects. The animals weighed 250 to $450 \mathrm{~g}$ and were housed in male/female pairs in large cages ( $2 \mathrm{~m}$ long, $1.3 \mathrm{~m}$ wide, and $2 \mathrm{~m}$ high). Temperature measurements were performed at the Primate Center, University of Brasília, DF, Brazil, where the animals were kept. Thirteen subjects were born in captivity and 11 subjects were of unknown origin, but all of them had lived in captivity for at least 9 months. They were fed a variety of foods and water was available ad libitum. The experimental procedures and housing conditions were in accordance with the principles and regulations of the Ethics Committee of the University of Brasília for the care and use of animals for scientific purposes.

\section{Procedure}

Temperature measurements were made in degrees Celsius with a self-calibrating thermometer (ThermoScan, Braun, Cologne, Germany). All temperature measurements were made in the subjects' home cages between 7:30 and 10:00 am. The caretaker, using a net, captured each subject and restrained it firmly for measurement. Two TM temperatures were obtained for each ear at 30-s intervals. The experimenter recorded the mean temperature reading. Order of subjects and ear testing were randomized. The whole procedure took place with minimum conversation and simple gestures to minimize the animals' excitement.

\section{Data analysis}

Differences between left and right TM temperature were analyzed by the paired signed ranks Wilcoxon test. In order to examine whether manipulation history, which means how many times each subject was captured during captivity, could be affecting the TM temperature, the Spearman two-tailed correlation test was applied to determine the correlation between number of previous captures and left and right TM temperatures. As the correlation test suggested the influence 
of previous experience, the animals were divided into two groups: "low capture history" group (LCH) submitted to 1 to 4 previous captures, and "high capture history" group $(\mathrm{HCH})$ submitted to 5 to 9 previous captures in captivity. The two-tailed MannWhitney U-test was used to compare left $v s$ right values in the $\mathrm{LCH}$ and $\mathrm{HCH}$ groups separately and to determine differences between left and right TM temperatures between the $\mathrm{LCH}$ and $\mathrm{HCH}$ groups. The data for each group are reported as means $\pm \mathrm{SEM}$. We set $\alpha$ at 0.05 for all analyses.

\section{Results}

There were no significant differences between gender (right temperature: $\mathrm{N}_{\text {males }}=$ $14,38.76 \pm 0.18^{\circ} \mathrm{C} ; \mathrm{N}_{\text {females }}=10,38.3 \pm$ $0.41^{\circ} \mathrm{C} ; \mathrm{P}=0.208$; left temperature: $\mathrm{N}_{\text {males }}=$ $14,39.07 \pm 0.26^{\circ} \mathrm{C} ; \mathrm{N}_{\text {females }}=10,38.2 \pm$ $\left.0.43^{\circ} \mathrm{C} ; \mathrm{P}=0.905\right)$. Therefore, data for males and females were pooled into one group. Comparisons of the temperatures measured in all 24 subjects of this study indicated no significant differences between left and right TM temperatures $(\mathrm{P}=0.457$; Wilcoxon test $)$.

It is known that with successive captures carried out with appropriate procedures, laboratory animals tend to show decreased stressrelated responses (10). Our subjects had previously been captured for routine veterinary procedures in the Primate Center, University of Brasília. Therefore, we decided to determine whether manipulation history could influence the tympanic temperature. Table 1 shows the tympanic temperature of the $\mathrm{LCH}$ and $\mathrm{HCH}$ groups. When we correlated the left and right TM temperatures with the number of previous captures, a significant negative correlation emerged for the right ear $(r=$ $-0.633 ; \mathrm{P}<0.001$; Spearman correlation test). No correlation was found for the left ear. Subjects that had a greater number of previous captures presented a lower temperature in the right ear (see Table 1 and Figure 1). Post hoc comparison of left $v s$ right values in the $\mathrm{LCH}$ and $\mathrm{HCH}$ groups separately showed no significant difference ( $\mathrm{P}>0.50$; MannWhitney U-test). However, comparison of TM temperatures between the $\mathrm{LCH}$ and $\mathrm{HCH}$ groups indicated that $\mathrm{HCH}$ subjects showed a lower right ear TM temperature $(\mathrm{P}<0.001$; Mann-Whitney U-test; Figure 2). There were no significant differences in left ear TM temperatures between the two groups $(\mathrm{P}=$ 0.173; Mann-Whitney U-test; Figure 2).

Table 1. Right and left ear tympanic temperature (mean \pm SEM) of marmosets from the low capture history group $(N=13)$ and high capture history group $(N=11)$.

\begin{tabular}{cccccc}
\hline \multirow{2}{*}{ Number } & \multicolumn{3}{c}{ LCH } & & \multicolumn{2}{c}{$\mathrm{HCH}$} \\
\cline { 2 - 3 } \cline { 5 - 6 } & Left ear & Right ear & Left ear & Right ear \\
\hline 1 & $39.20 \pm 0.15$ & $40.25 \pm 0.29$ & & $35.75 \pm 0.06$ & $38.45 \pm 0.23$ \\
2 & $39.80 \pm 0.07$ & $39.60 \pm 0.50$ & & $40.05 \pm 0.10$ & $38.25 \pm 0.19$ \\
3 & $39.90 \pm 0.00$ & $39.40 \pm 0.11$ & & $39.55 \pm 0.06$ & $38.10 \pm 0.25$ \\
4 & $39.00 \pm 0.07$ & $38.85 \pm 0.25$ & & $37.65 \pm 0.31$ & $38.50 \pm 0.42$ \\
5 & $38.95 \pm 0.72$ & $40.35 \pm 0.01$ & $39.40 \pm 0.08$ & $38.65 \pm 0.19$ \\
6 & $37.55 \pm 0.21$ & $38.40 \pm 0.11$ & $38.50 \pm 0.08$ & $36.25 \pm 0.06$ \\
7 & $38.85 \pm 0.29$ & $38.80 \pm 0.03$ & $36.80 \pm 0.00$ & $37.30 \pm 0.29$ \\
8 & $39.45 \pm 0.01$ & $40.10 \pm 0.03$ & $36.20 \pm 0.08$ & $37.40 \pm 0.46$ \\
9 & $37.80 \pm 0.03$ & $38.20 \pm 0.15$ & $38.30 \pm 0.00$ & $37.75 \pm 0.06$ \\
10 & $38.95 \pm 0.13$ & $39.05 \pm 0.29$ & $40.00 \pm 0.08$ & $38.40 \pm 0.34$ \\
11 & $39.85 \pm 0.01$ & $38.75 \pm 0.29$ & $38.05 \pm 0.36$ & $37.10 \pm 0.21$ \\
12 & $40.00 \pm 0.11$ & $38.95 \pm 0.17$ & & \\
13 & $39.55 \pm 0.17$ & $38.80 \pm 0.11$ & &
\end{tabular}

LCH, low capture history group,1-4 captures; $\mathrm{HCH}$, high capture history group, 5-9 captures.

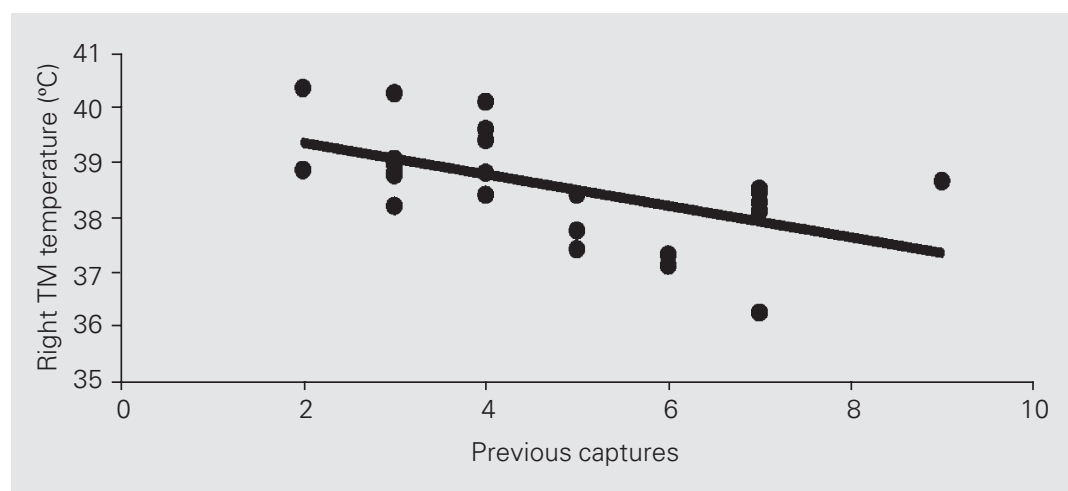

Figure 1. Effect of number of previous captures on tympanic membrane (TM) temperature in the right ear of Callithrix penicillata marmosets during captivity $(N=24)$. There was a significant negative correlation between the TM temperature of the right ear and the number of previous captures ( $r=-0.633 ; \mathrm{P}<0.001$; Spearman correlation test). 


\section{Discussion}

The major finding of the present study is that marmosets showed differences between left and right ear TM temperatures in a stressful situation. This difference was the result of the previous experience of the subjects in relation to the paradigm employed in this study. That is, subjects with a longer history of stressful contention showed lower right TM temperature when compared to subjects with a short history of captures and contention. No difference was observed for TM temperature recorded in the left ear. These data suggest a brain hemispheric specialization in marmosets, with the right hemisphere being related to biobehavioral stress responses.

Subjects with 5 to 9 previous captures ( $\mathrm{HCH}$ group) presented a lower temperature in the right TM, suggesting a possible asymmetric rise in metabolic activity of that cerebral hemisphere. A number of studies have suggested that the right hemisphere is spe-

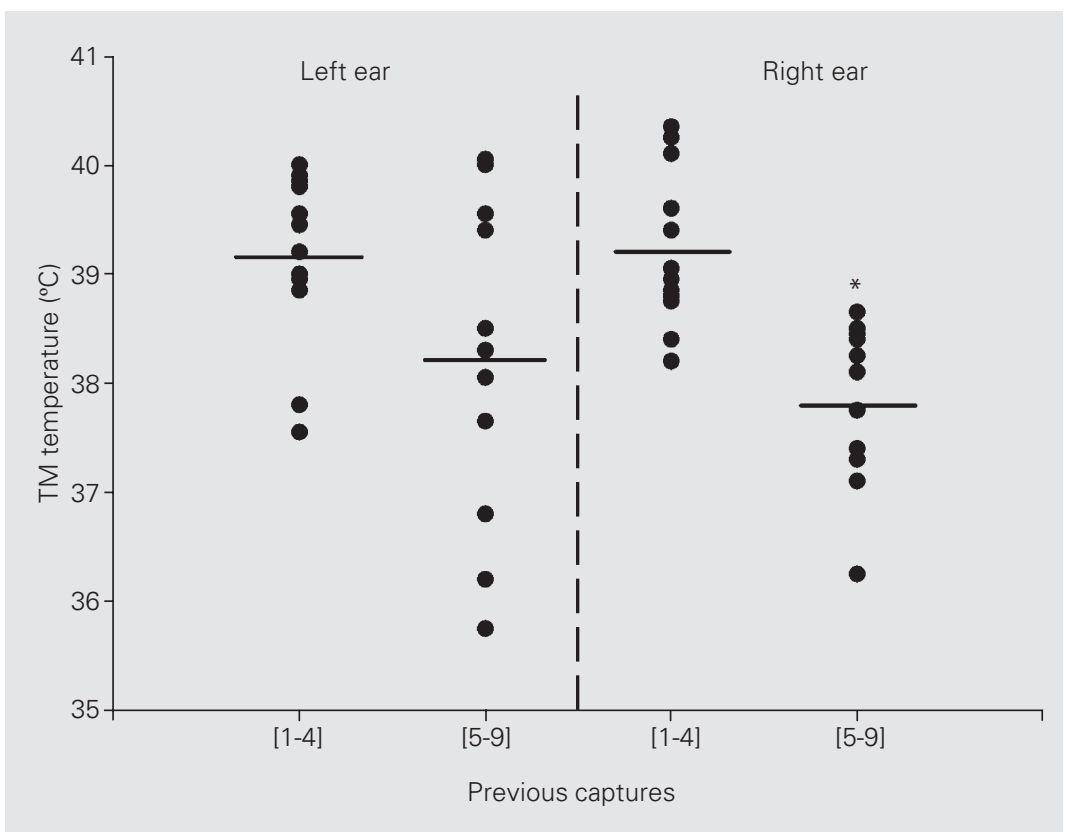

Figure 2. Left and right ear tympanic membrane (TM) temperature in Callithrix penicillata related to the number of previous captures. Each point represents one marmoset $(N=24)$. The horizontal bars indicate the mean temperature. ${ }^{*} \mathrm{P}<0.001$ compared to right ear $(1-4$ previous captures) (Mann-Whitney U-test). cialized for processing emotional information and stress-related responses (e.g., 1113). It is possible that these animals underwent a sensitization process as they were captured, while those with a smaller number of previous captures had a less intensive activity that was not strong enough to create an adequate amount of blood flow to significantly cool the right hemisphere. On the other hand, we cannot rule out the possibility of a habituation process, because it is known that skilled and frequent manipulations can reduce stress during physical restraint of captive animals (10).

Our results agree with those reported by Boyce et al. (5) in a study examining a possible association between left-to-right TM temperature asymmetries and biobehavioral stress responses in rhesus macaques and children. These investigators found cooler right ear TM temperature, suggesting that the right hemisphere was more involved in the stress response.

The extreme excitement of the animals during capture, with activation of structures of the right hemisphere, resembles the activation of the right ventral prefrontal cortex observed by magnetic resonance imaging in human patients during intervals between panic attacks, when anticipatory anxiety prevails (14). In addition, in normal subjects, anxiety induced by expectation of painful electric stimuli increases blood flow in the right amygdala (14).

The emotional response of marmosets is characterized by a range of complex behaviors $(15,16)$ and physiological changes $(17)$. It is believed that plasticity has been modeled in marmosets along evolution due to the strong predation pressure suffered by this little anthropoid (18). Phylogenetic trees based on molecular markers have shown that marmosets diverged from the ancestral primate about 40 million years ago (19). Brain lateralization could refine emotional responses, which are advantageous during defensive behavior. According to the function 
hierarchization theory (20), in an extremely stressful situation such as capture and restraint, activation of neurofunctional sidelong dominant areas can be advantageous when postponing other emotional and cognitive functions until a more favorable opportunity, avoiding competition of functions.

Taken together, the results of the present study show a lateralized emotional response to capture and restraint which depends on the previous experience of the subject. Furthermore, they indicate that the procedure used in this study is a reliable technique for indirect assessment of brain hemispheric activity in primates. To our knowledge, this is the first report of laterality in the physiological processing of biobehavioral stress responses in a New World monkey (Callithrix penicillata). Additional studies are necessary in order to gain more direct information about lateralized control of emotional processing in marmosets.

Indeed, the evidence presented here is not unequivocal. There is a lack of knowledge concerning the anatomy of tympanic and cerebral blood flow in marmosets that could be related to changes during stressful situations. More direct evidence using electroencephalogram analysis, interbrain electrode stimulation, inhibition of powerful vasodilators such as $\mathrm{NO}$ and $\mathrm{CO}_{2}$, and anxiolytic drugs could help to better understand these aspects. It should also be pointed out that there were marked individual differences among subjects and that by increasing the number of individuals we may find subpopulations with higher emotional reactivity, indicating (or not) an accentuated picture of emotional lateralization. This last step is being investigated now at the Primate Center, University of Brasília.

\section{Acknowledgments}

We thank Gustavo Canale for assistance with data collection and Dr. Raimundo Oliveira for animal care. We are grateful to two anonymous reviewers for helpful comments.

\section{References}

1. Fagot J \& Vauclair J (1991). Manual laterality in nonhuman primates: a distinction between handedness and manual specialization. Psychological Bulletin, 109: 76-89.

2. MacNeilage PF, Studdertkennedy MG \& Lindblom B (1987). Primate handedness reconsidered. Behavioral and Brain Sciences, 10: 247263.

3. Hook-Costigan MA \& Rogers LJ (1998). Lateralized use of the mouth in production of vocalizations by marmosets. Neuropsychologia, 36: 1265-1273.

4. Kosslyn SM, Gazzaniga MS, Galaburda AM \& Rabin C (1999). Hemispheric specialization. In: Zigmond MJ, Bloom FE, Landis SC, Roberts JL \& Squire LR (Editors), Fundamental Neuroscience. Academic Press, San Diego, CA, 1531-1542.

5. Boyce WT, Hihley JD, Jeremin JJ, Champoux M \& Suomi SJ (1996). Tympanic temperature asymmetry and stress behavior in rhesus monkeys and children. Archives of Pediatrics and Adolescent Medicine, 150: 518-523.

6. Hopkins WD \& Fowler LA (1998). Lateralized changes in tympanic membrane temperature in relation to different cognitive tasks in chimpanzees (Pan troglodytes). Behavioral Neuroscience, 112: 8388.

7. Meiners ML \& Dabbs JM (1977). Ear temperature and brain blood flow: laterality effects. Bulletin of the Psychonomic Society, 10: 194-
196.

8. Parr LA \& Hopkins WD (2000). Brain temperature asymmetries and emotional perception in chimpanzees, Pan troglodytes. Physiology and Behavior, 71: 363-371

9. Swift AB (1991). Tympanic thermometry: an index of hemispheric activity. Perceptual and Motor Skills, 73: 275-293.

10. Kohn FB (1992). Regular weight monitoring as a management and research tool. Animal's Keeper Forum, 19: 173-181.

11. Borod JC \& Madigan NK (2000). Neuropsychology of emotion and emotional disorders: an overview and research directions. In: Borod JC (Editor), The Neuropsychology of Emotion. Oxford University Press, New York, 3-28.

12. Davidson RJ \& Irwin W (1999). The functional neuroanatomy of emotion and affective style. Trends in Cognitive Sciences, 3: 11-21.

13. Wittling $W$ (1997). The right hemisphere and the human stress response. Acta Physiologica Scandinavica, 161: 55-59.

14. Drevets WC \& Raichle ME (1995). Positron emission tomographic imaging studies of human emotional disorders. In: Gazzaniga MS (Editor), The Cognitive Neurosciences. MIT Press, Cambridge, MA, 1153-1164

15. Barros M, Boere V, Huston JP \& Tomaz C (2000). Measuring fear and anxiety in the marmoset (Callithrix penicillata) with a novel predator confrontation model: effects of diazepam. Behavioural Brain 
Research, 108: 205-211.

16. Stevenson MF \& Rylands $A B$ (1988). The marmosets, genus Callithrix. In: Mittermeier RA, Rylands AB, Coimbra-Filho AF \& Fonseca GAB (Editors), Ecology and Behavior of Neotropical Primates. Vol. 2. World Wildlife Fund, Washington, DC, 131-222.

17. Saltzman W, Prudom SL, Schultz-Darken J \& Abbott DH (2000). Reduced adrenocortical responsiveness to adrenocorticotropic hormone (ACTH) in socially subordinate female marmoset monkeys. Psychoneuroendocrinology, 25: 463-477.
18. Ford SM (1980). Callitrichids as phyletic dwarfs and the place of the Callitrichidae in Platyrrhini. Primates, 21: 31-43.

19. Kageyama $T$ (2000). New World monkey pepsinogens $A$ and $C$, and prochymosis. Purification, characterization of enzymatic properties, cDNA cloning, and molecular evolution. Journal of Biochemistry, 127: 761-770

20. Galaburda AM, Rosen GD \& Sherman GF (1990). Individual variability in cortical organization - its relationship to brain laterality and implications to function. Neuropsychologia, 28: 529-546. 